\title{
A Laser Diode-Based Wireless Optogenetic Headstage
}

\author{
Alireza Mesri \\ Department of Electronics, Information \\ and Bioengineering (DEIB) \\ Politecnico di Milano \\ Milan, Italy \\ alireza.mesri@polimi.it \\ Marco Sampietro \\ Department of Electronics, Information \\ and Bioengineering (DEIB) \\ Politecnico di Milano \\ Milan, Italy \\ marco.sampietro@polimi.it
}

\author{
André B. Cunha \\ Department of Physics \\ University of Oslo \\ Oslo, Norway \\ andre.cunha@fys.uio.no \\ Giorgio Ferrari \\ Department of Electronics, Information \\ and Bioengineering (DEIB) \\ Politecnico di Milano \\ Milan, Italy \\ giorgio.ferrari@polimi.it
}

\author{
Ørjan G. Martinsen \\ ${ }^{1}$ Department of Physics \\ ${ }^{2}$ Department of Clinical and Biomedical \\ Engineering \\ ${ }^{1}$ University of Oslo, ${ }^{2}$ Oslo University \\ Hospital \\ Oslo, Norway \\ ogm@,fys.uio.no
}

\begin{abstract}
A power efficient, battery powered optogenetic headstage for doing in-vivo experiments with freely moving genetically modified animals is presented. The proposed system is designed with commercial off-the-shelf components, and is based on a Bluetooth Low Energy (BLE) System-on-Chip (SoC) with an integrated antenna and a programmable ARM Cortex-M3 microprocessor core able to control the circuit. The optical signal is generated using a compact laser diode (LD) suitable for a wearable headstage. LD produces light in a highly concentrated way considerably improving the LD-optical fiber coupling efficiency. The proposed optogenetic system is shown to provide $120 \mathrm{~mW} / \mathrm{mm}^{2}$ at the fiber tip with a current consumption of 60mA, considerably lower than LED-based systems. The system is remotely controlled by a smartphone app where the user can define optical stimulations patterns settings (optical power, frequency, duty cycle, etc.). It is also powerful enough to be ready to house additional optogenetics functionalities, like electrochemical sensing of the cell response, without significant modifications, thus being the basis of an integrated optogenetic platform.
\end{abstract}

Keywords-optogenetics, laser diode, optical stimulation, headstage, channelrhodopsin

\section{INTRODUCTION}

Optogenetics, first introduced in 2005 [1], is a ground breaking biomedical technique, which combines optics and genetics to control the activity of cells at in vitro and in vivo conditions. It is based on introducing opsins, light sensitive proteins, into neurons through genetic engineering approaches. Optogenetics has emerged as a tool that enables the activation or inhibition of specific cell populations in the brain by the use of different wavelengths of light. For excitatory Channelrhodopsins, and inhibitory Halorhodopsins, blue and yellow wavelengths are needed, respectively[2,3].

The stimulation of deep brain areas is necessary for treatment of some neurodegenerative disorders, like Parkinson

The research is under Training4CRM project which is funded by the European Union Horizon 2020 Programme (H2020-MSCA-ITN-2016) under the Marie Skłodowska-Curie Initial Training Network and Grant Agreement No.722779. Project website : www.training4crm.eu
Disease (PD), and Epilepsy (EPI). Electrical deep brain stimulation (DBS) affects all cells in the stimulated area in an indiscriminative way and the treatment loses efficacy over time. Also, confounding thermal effects, mood alteration, motor and sensory control issues or depression are some of side effects derived from the application of that method [4]. In contrast, optogenetic allow us to stimulate genetically modified cells in a more precise and selective way, and can address those challenges $[1,2,4,5]$. In the framework of the European Union project "Training4CRM", we are developing a platform to exploit the potential of optogenetics for the treatment of PD and EPI [6].

Channelrhodopsin-2 (ChR2), the main optogenetic protein, is introduced to modulate neuronal activities [1]. For the activation of ChR2, blue light in the spectral range from 440 to $490 \mathrm{~nm}$ is needed, operating on the millisecond timescale with $1 \mathrm{~mW} / \mathrm{mm}^{2}$ optical power. Higher power source should anyhow be used due to the strong light absorption by the brain tissue. As an example, for $473 \mathrm{~nm}$ wavelength, the intensity of light inside the brain tissue will drop to about $1 \%$ after $1 \mathrm{~mm}$ of travelling $[4,5]$.

Different light-delivery methods have been suggested and are used for the manipulation of neuronal activity. LEDs have become progressively interesting for optogenetic experimentation in freely moving animals. Low cost, small dimension, reliability, and stability are some of their advantages. But, one serious disadvantage of LEDs is poor LED-fiber coupling[2]. Different methods are proposed to improve LED fiber coupling [7-9]. In [7], authors proposed silicon housing technique to improve LED-fiber coupling, achieving $1.71 \mathrm{~mW} / \mathrm{mm}^{2}$ with a driving current of $30 \mathrm{~mA}$. In [8], they have suggested a micro-lens coupled LED, which improves light intensity by $99 \%$. All these techniques, need specific fabrication approaches. In [9], the LED is coupled to an implanted $200 \mu \mathrm{m}$ diameter optical fiber. For a $150 \mathrm{~mA}$ LED current, $70 \mathrm{~mW} / \mathrm{mm}^{2}$ is achieved. 
A laser light source can deliver light with low divergence improving the coupling with the optical fiber, and is able to activate genetically modified neurons within millimeters of the fiber. However, the laser-based systems reported in the literature use a bulky laser source with a long fiber from the lab bench to the animal. Therefore, it tethers the animals, and restricts their natural behavior[1,5].

Here, we report a new power-efficient wireless optogenetic headstage based on a laser diode (LD) to provide the optical power for stimulation, which significantly reduces the required current for a given optical power in comparison with previously reported works. With $60 \mathrm{~mA}$ stimulation current, it can provide $120 \mathrm{~mW} / \mathrm{mm}^{2}$ at the fiber tip. It also features a Bluetooth Low Energy (BLE) which allows the adjustment of the optical power stimulation through any a BLE enabled client (smartphone, tablet, PC, smartwatches).

The paper is organised as follows. The proposed optogenetic system is presented in Section II. Section III presents measurement results, which validate the performance of the platform. Comparisons with other methods from the literature are reported in Section IV. Finally, section V summarizes the features of the proposed optogenetic system.

\section{SYSTEM DESCRIPTION}

\section{A. Hardware}

Fig. 1 shows the optogenetic system structure. We have developed the optogenetic circuit in two boards. PL 450B, an OSRAM blue LD, is used as a light source for the optogenetic system. $3.8 \mathrm{~mm}$ package size, $450 \mathrm{~nm}$ emission wavelength, and $80 \mathrm{~mW}$ power are the main features of the LD. At $25^{\circ} \mathrm{C}$, with $24 \mathrm{~mA}$ driving current, it can provide an optical power of about $11 \mathrm{~mW}$ [10].

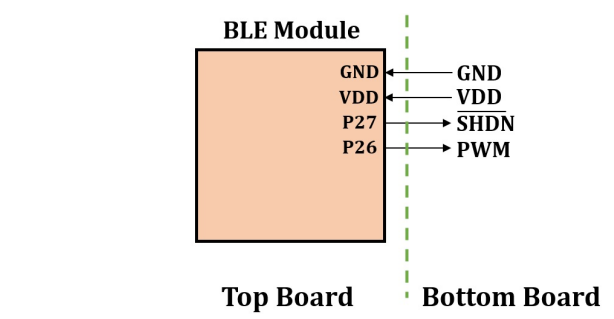

(a)

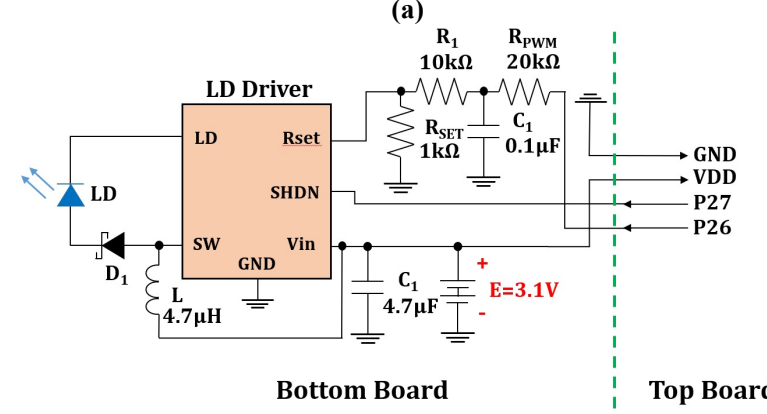

(b)

Fig. 1. Schematic of the proposed optogenetic headstage: (a) Top board, and (b) Bottom board.
The headstage consists of two interconnected boards. The top board (Fig. 1a) is dedicated for the BLE System-on-Chip (SoC). The board will be connected through 4 pins to the bottom board, which has the LD, the LD driver IC, and two batteries in series to provide $3.1 \mathrm{~V}$ supply voltage. Two pins (VDD, GND) provide supply voltage from batteries in the bottom board for the BLE SoC. The other two pins provide the stimulation pattern, and shutdown (SHDN) signal for the LD driver IC. Fig. 1b shows the schematic for the LD driver stage. SHDN pin allow us to turn on the LD driver IC during stimulation. $\mathrm{R}_{\mathrm{SET}}$ defines the maximum current at the LD pin.

Anaren's BLE SoC A20737 was selected to control the circuit. It is based on an ARM Cortex-M3 microprocessor core and features an integrated antenna facilitating the PCB design [11]. This programmable SoC includes a non-changeable kernel which accepts instructions written on an extended application programming interface (API) of the C programming language. This kernel provides an event-driven architecture on top of which Anaren has built an abstraction layer API which allows loop based programming (Atmosphere). This API takes care of the entire BLE stack in both client (smartphone) and server (optogenetic implant) and simplifies numerous tasks. It works in $2.4 \mathrm{GHz}$ industrial, scientific and medical (ISM) frequency band, and its package size is $11 \times 13 \times 2.5 \mathrm{~mm}^{3}$. The weight is around 0.5 grams and can work from 1.7 to $3.6 \mathrm{~V}$ power supply voltages. Its average current consumption during sleep and no sleep modes is $1.5 \mathrm{~mA}$, and $4.5 \mathrm{~mA}$, respectively. Pin 26 , which provides the PWM signal with the programmable frequency and duty cycle is used to generate the stimulation pattern. Pin27, is used to turn on the LD driver IC during stimulation.

We have selected LT1932 from Linear Technology as the LD driver. It is a constant frequency step-up DC/DC converter which operates as a constant-current source. The value of $R_{\mathrm{SET}}$ defines the LD current between $5 \mathrm{~mA}$ and $40 \mathrm{~mA}$. There are different methods to change the current value of the LD pin. We have applied the PWM signal to the $\mathrm{R}_{\mathrm{SET}}$ pin in order to change the current of the LD pin. In this case, increasing the duty cycle of the PWM signal will reduce the LD current and thus its brightness [12].

The power supply is provided by two Zinc/monovalent Silver Oxide batteries SR927W, from Renata company. They have a nominal voltage of $1.55 \mathrm{~V}$, a capacity of $55 \mathrm{mAh}$ and a weight as low as $0.77 \mathrm{~g}$. A solution with a single battery and a $\mathrm{DC} / \mathrm{DC}$ converter to increase the voltage was discarded due to the excessive increase of the current absorption during the optical stimulation.

\section{B. Software}

The proposed firmware is built on the loop structure Anaren provides. It is designed as a finite state machine using a control counter which also functions as the main timer for the system (see Fig. 2). 


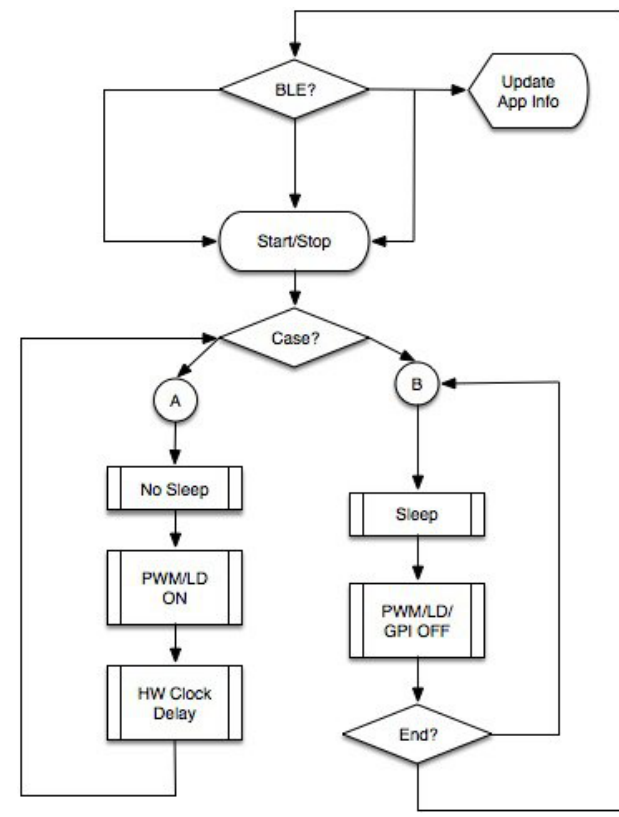

Fig. 2. Flowchart for system firmware.

The structure of the finite state machine is based on a nested if/else structure which being a basic $\mathrm{C}$ structure, translates itself into less machine code which results in less computational power while being more reliable as proven by other tested alternatives. To ensure PWM precision without compromising power efficiency, the SoC is set on sleep mode only during dead periods. BLE notifications are only broadcasted while connected through BLE. To ensure reliability, no stimulation periods are a $0 \%$ duty cycle PWM rather than disabling it. The app is developed through Anaren software tools which generate a Cordova source portable to both Android and iOS.

\section{MEASUMENT RESUlts}

Fig. 3 shows a preliminary prototype of the proposed optogenetic headstage. Dimension of the headstage is $25 \times 20 \times 17 \mathrm{~mm}^{3}$, and its weight is 6.8 grams where 1.5 grams is due to 2 batteries. We have designed the prototype using the BLE-Anaren A20737-KD Circuits which has access to all pins, 2 buttons and a LED for programming and testing purposes.

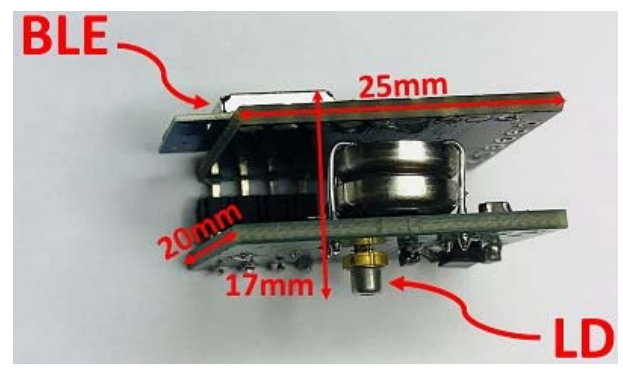

Fig. 3. Proposed optogenetic headstage
To investigate the performance of the headstage, we have emulated a standard optogenetic experiment by applying 150 pulses at $10 \mathrm{~Hz}$ every 6 minutes, repeated for 40 times (4 hours). Duty cycle of $10 \mathrm{~Hz}$ pulses is $5 \%$ generating stimulation pulses of $5 \mathrm{~ms}$. During the stimulation time, it is possible to change the optical stimulation power by changing the duty cycle in the app. The PWM signal frequency, and the duty cycle were selected to be $5 \mathrm{kHz}$, and $5 \%$, respectively.

The optical power at the tip of a $200 \mu \mathrm{m}$ diameter optical fiber (Thorlabs-CFMXB20) was measured using a Centronic Series 5T photodiode coupled to DHPCA-100 variable gain transimpedance amplifier (TIA). The photodiode was calibrated using the digital optical meter PM110D by Thorlabs. For $450 \mathrm{~nm}$ as a wavelength of $\mathrm{LD}$, the photo diode responsivity is about $0.25 \mathrm{~A} / \mathrm{W}$. The output optical power at the fiber tip is equal to :

$$
\text { Pout }_{\text {opt. }}=\alpha \text { Vout } /(0.25 \times 100)
$$

Where Vout is the output voltage of the TIA, and $100 \Omega$ is its gain. Also, $\alpha$ is the optical power level dependent parameter, and is obtained by calibration. It should be mentioned that the coupling efficiency of the LD-fiber is about $45 \%$. Due to LD's package which makes coupling of the cannula to light source simpler, getting higher than $35 \%$ is easily achievable, while we achieved about $15 \%$ for 0402 package LEDs, which also generate much lower optical power.

Fig. 4 shows the total current consumption of the headstage and the measured TIA output voltage of a single optical pulse. During stimulation, the current consumption is about $65 \mathrm{~mA}$, where $60 \mathrm{~mA}$ is given by the LD driver circuit and the LD. The output voltage of the TIA is about $80 \mathrm{mV}$. By considering the photodiode sensitivity, the optical power at the fiber tip is $3.77 \mathrm{~mW}\left(120 \mathrm{~mW} / \mathrm{mm}^{2}\right)$.

\section{COMPARISON}

Table I provides a comparison between the proposed optogenetic stimulation headstage and other recent works. Programmability, current consumption, and optical power at the fiber tip are the main features of the stimulation stage.

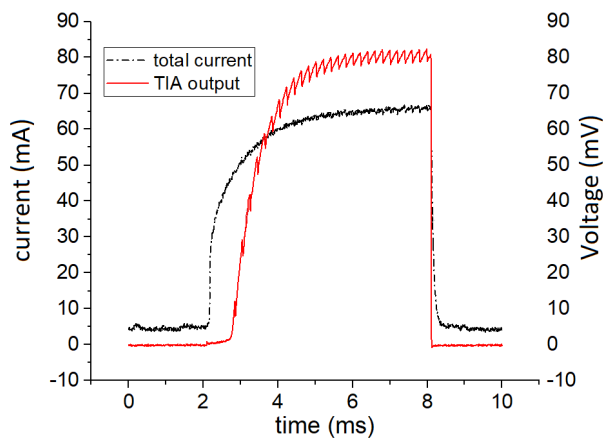

Fig. 4. Total current of the headstage (left axis) and TIA output voltage (right axis) 
TABLE I. COMPARISON TABLE

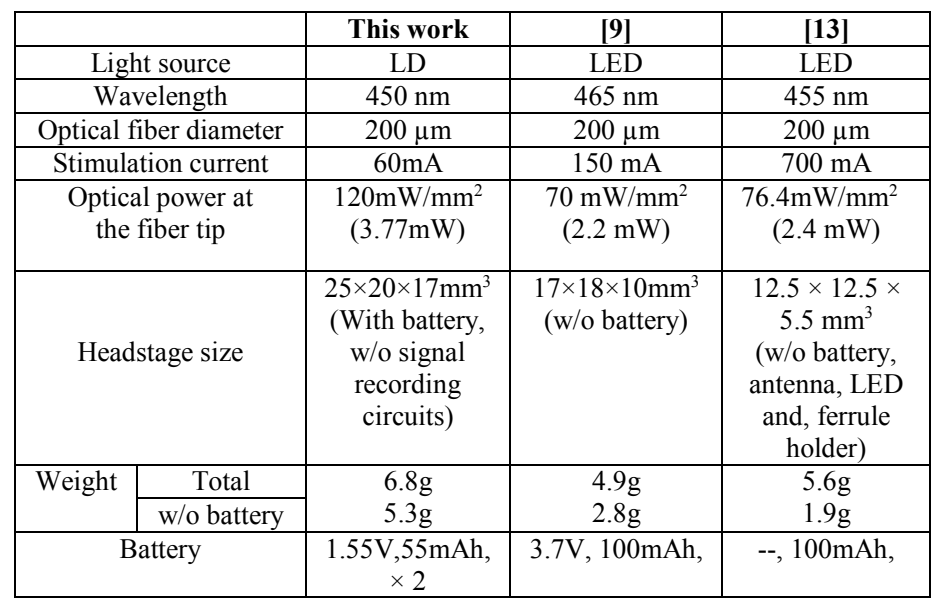

All systems are based on the coupling of the blue light source to an optical fiber which delivers the light into the area of interest in the brain. Also, they are using the battery to power up the system, and they can be mounted directly on the head of the animal. Based on the provided data in the table, while our circuit is using much less current in comparison with other systems, it provides the highest optical power at the fiber tip. Light emission of the LD in a highly concentrated way is the main reason for such considerable improvement. It should be mentioned that in the reported headstage in [9], which performs both optical stimulation and electrophysiological recording (with 32 recording channels) simultaneously, about $48 \%$ of total power consumption is related to stimulation stage. Size of the proposed headstage is bigger than previous works. But it is based on the BLE board provided by KD Circuits, which has been designed to consider the usage of all pins and functions of the BLE SoC. Therefore, by designing new board as well as ordering a lower thickness for the FR4 layer of the $\mathrm{PCB}$, not only we can reduce the size of the boards, but also the weight of the system.

\section{CONCLUSIONS AND FUTURE WORKS}

In this paper, a new power efficient battery powered optogenetic system is proposed based on only commercial offthe-shelf components. Thanks to the LD as a light source, significant improvements in terms of optical power and current consumption are achieved. It can provide $120 \mathrm{~mW} / \mathrm{mm}^{2}$ at the fiber tip while it consumes a current of $60 \mathrm{~mA}$. Also, due to the selected structure and the BLE SoC, the users can control the system and the optical power of the stimulation pattern through the app. Finally, by using a PWM signal to adjust the optical power for stimulation, the circuit complexity is reduced.

The next step is to do in-vitro and in-vivo experiments with modified version of the headstage. Moreover, the functionalities of the headstage will be enlarged including the possibility to perform electrochemical measurements to sense the neurotransmitter releases stimulated by the optical signal. The BLE SoC has been already selected powerful enough to manage cyclic voltammetry, and amperometry. For this purpose, we will use the ADC of the BLE SoC to measure and send data to a platform(smart device, cloud, PC). Also, a SPI bus will be used to program an external DAC in order to produce the required signals for doing electrochemical measurements. We will use a carbon based optoelectrical waveguide to deliver both light to the brain and doing electrochemical measurements which will correspond to the released dopamine.

\section{ACKNOWLEDGMENT}

The authors would like to thanks Prof. Merab Kokaia, and Dr. Fredrik Berglind at Epilepsy Center of Lund University for their useful discussion about the optogenetic systems and experiments, and Prof. Jenny Emnéus for leading the Training4CRM project.

\section{REFERENCES}

[1] N. Li, and P. Miao, "Let there be light: A tutorial on optogenetics," in IEEE Pulse, vol. 5, no. 4, pp. 55-59, July-Aug. 2014.

[2] M. R. Warden, J. A. Cardin, and K. Deisseroth, "Optical neural interfaces,” Annu. Rev. Biomed. Eng., vol. 16, pp. 103-29, Jul. 2014.

[3] H. Zhao, "Recent progress of development of optogenetic implantable neural probes,” Int. J. Mol. Sci., vol. 18, no. 8, pp. 1-21, Aug. 2017.

[4] R. Pashaie, P. Anikeeva, J. H. Lee, R. Prakash, O. Yizhar, et al., "Optogenetic brain interfaces," in IEEE Reviews in Biomedical Engineering, vol. 7, pp. 3-30, 2014.

[5] M Aravanis, L. P.Wang, F. Zhang, L. A. Meltzer, M. ZMogri, et al., "An optical neural interface: in vivo control of rodent motor cortex with integrated fiberoptic and optogenetic technology,"J. Neural Eng., vol 4, no.3, pp. S143-S156, Sep. 2007.

[6] Training4CRM project. Available online : http://www.training4crm.eu/

[7] M. Schwaerzle, P. Elmlinger, O. Paul and P. Ruther, "Miniaturized tool for optogenetics based on an LED and an optical fiber interfaced by a silicon housing," 2014 36th Annual International Conference of the IEEE Engineering in Medicine and Biology Society, Chicago, IL, pp. 5252-5255, 2014.

[8] X. Bi, T. Xie, B. Fan, W. Khan, Y. Guo and W. Li, "A flexible, microlens-coupled LED stimulator for optical neuromodulation," in IEEE Transactions on Biomedical Circuits and Systems, vol. 10, no. 5, pp. 972-978, Oct. 2016.

[9] G. Gagnon-Turcotte, Y. LeChasseur, C. Bories, Y. Messaddeq, Y. De Koninck and B. Gosselin, "A wireless headstage for combined optogenetics and multichannel electrophysiological recording," in IEEE Transactions on Biomedical Circuits and Systems, vol. 11, no. 1, pp. 114, Feb. 2017.

[10] OSRAM PL 450B laser diode. Available online https://www.osram.com/os/ecat/TO38\%20PL\%20450B/com/en/class pi m web catalog 103489/global/prd pim device 2220052/

[11] Anaren Integrated Radio(AIR). Available online : https://app.atmosphereiot.com/wiki/A20737_Module

[12] Linear Technology. LT1932 Constant-Current DC/DC LED Driver in ThinSOT. Available online http://cds.linear.com/docs/en/datasheet/1932f.pdf

[13] Multichannel systems, w2100-hs4-opto. Available online https://www.multichannelsystems.com/products/w2100-hs4-opto 